\title{
Turbulence reduction in a boundary layer by a local spanwise oscillating surface
}

\author{
F. Laadhari, L. Skandaji, and R. More ${ }^{\text {a) }}$ \\ Laboratoire de Mécanique des Fluides et d'Acoustique, Ecole Centrale de Lyon-Université Claude Bernard- \\ URA CNRS 263, BP 163, 69131 Ecully, France
}

(Received 8 March 1994; accepted 1 June 1994)

\begin{abstract}
This Letter describes an experimental investigation of the response of a turbulent boundary layer on a flat plate to a local spanwise oscillation of the wall, with a nondimensional frequency $f^{+}$varying between 0.0033 and 0.0166 . The investigation has been carried out for a wall motion amplitude $\Delta z^{+}=160$. The three components of the turbulence intensities and the Reynolds stress prove to be a decreasing function of the frequency. This reduction affects almost the whole boundary layer in a cross section located at the middle of the oscillating wall. The mean streamwise velocity $\bar{U}$ is reduced throughout the region $y^{+}<30$. The velocity profiles exhibit a well-defined log region when plotted against $y$, nondimensionalized with the friction velocity of the unperturbed boundary layer. The weighted probability density functions of $u$ and $v$ exhibit an increase in intensities of wallward motion related to changes in the structure of the oscillatory flow. The fine structure of the turbulence is also affected by the spanwise oscillation as shown by the reduction of Taylor's microscale.
\end{abstract}

Both experiments and numerical simulations ${ }^{1-3}$ have provided evidence that an initially fully developed twodimensional (2-D) boundary layer subjected to a sudden spanwise pressure gradient exhibits a decrease of turbulent quantities such as the Reynolds shear stress and the turbulent kinetic energy. This reduction is limited in time to the transient period needed for the flow to reach a new 2-D steady state, characterized by a new direction and a higher Reynolds number. Thus, adding spanwise motion actually reduces turbulent levels. This somewhat surprising result confirms that the spanwise flow does not contribute to turbulence production, as shown by Jung et al. ${ }^{4}$ in a numerical simulation of a planar channel flow. These authors have also shown that when a spanwise oscillatory motion of the channel walls is applied to the flow, there is a sustained reduction in the Reynolds shear stress.

As previously suggested, ${ }^{3}$ this reduction is related to the fact that the interaction between longitudinal vortices and the streaky pattern of the flow nearer the wall, is modified by the spanwise motion. The streamwise vortices are convected in the spanwise direction, breaking the original streaks as they cross over them. Furthermore the effect of the longitudinal vorticity induced by the spanwise velocity gradient is an alteration of the structures already present in the 2-D boundary layer, a reduction of the burst-sweep activity and an increase of viscous dissipation. In the undisturbed 2-D flow, longitudinal vortices are positioned in such a way as to pump low speed fluid away from the low speed streaks lying near the wall and to inject high speed fluid wallward into the high speed streaks. This mechanism explains the high value of the streamwise wall shear stress on the downwash side of streamwise vortices, where high speed fluid is slowed down by the wall. It also explains the creation of bursts of low speed fluid in the buffer layer. In the excited turbulent boundary layer, this pattern is disrupted by the spanwise flow which shifts the vortices to a different position relative to the near-wall streaks. Longitudinal vortices thus inject high speed fluid into low speed streaks and low speed fluid into high speed ones, weakening the intensity of the streaks. ${ }^{3}$

The present experiment was designed to suppress turbulence by keeping the boundary layer in an unsteady threedimensional (3-D) state by a spanwise oscillation of a wall section. The experiment was performed in an open-circuit low speed blower tunnel with a $500 \times 500 \mathrm{~mm}$ working section (Fig. 1). To generate a boundary layer, a flat plate $(4.5 \mathrm{~m}$ long, $0.5 \mathrm{~m}$ wide and $25 \mathrm{~mm}$ thick) is rigidly mounted along the center line of the test section. Turbulence in the boundary layer was triggered by a trip wire (3 $\mathrm{mm}$ diameter) placed 3 $\mathrm{cm}$ from the leading edge. The free-stream turbulence level in the tunnel was $0.4 \%$. The oscillating plate $(1 \mathrm{~m}$ long, 0.7 $\mathrm{m}$ wide and $10 \mathrm{~mm}$ thick) is placed $2.5 \mathrm{~m}$ from the leading edge, inset into and flush with the fixed plate. The gaps between the two plates are $0.2 \mathrm{~mm}$ and are bridged by a Teflon joint. The sinusoidal oscillation was produced by a crankshaft system (Fig. 1) with an operating frequency in the range 2 to $10 \mathrm{~Hz} \quad\left(0.0033 \leqslant f^{+} \leqslant 0.0166\right.$, where $\left.f^{+}=f \nu / u^{* 2}\right)$. The amplitude of the moving wall in all experiments was $2.5 \mathrm{~cm}\left(\Delta z^{+}=160\right)$. Velocity measurements were made with fixed probes: a single hot wire probe, two parallel hot wires for the streamwise velocity and its normal



FIG. 1. Schematic diagram of the experimental setup. 


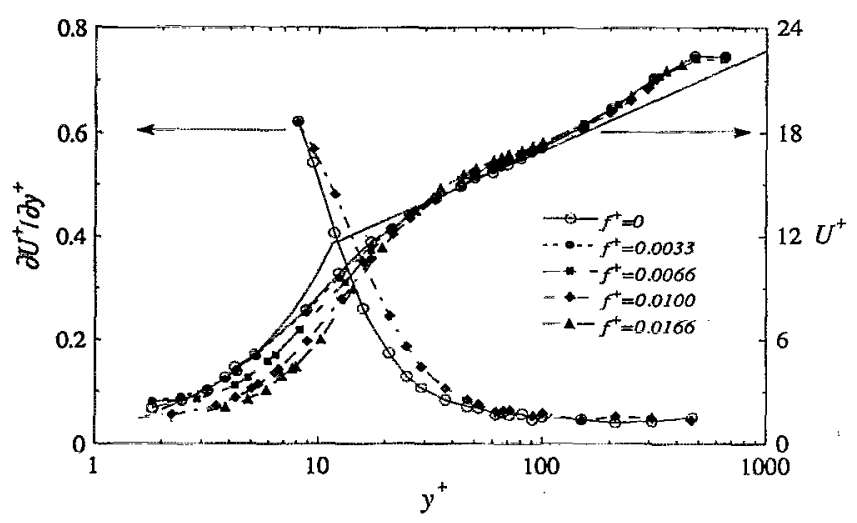

FIG. 2. Profiles of the mean streamwise velocity at different frequencies and its normal gradient at $f^{+}=0.01$, compared to the unperturbed boundary layer (open symbols).

gradient; $X$ and $v$ shaped probes for the normal and spanwise components, respectively. The signals from the hot wire anemometers were amplified and low-pass filtered at $2 \mathrm{kHz}$ before being routed to the data acquisition system and then digitized.

At the measurement station, $x=3 \mathrm{~m}$; the unperturbed boundary layer was two dimensional and was characterized by the following parameters: $\delta=69 \mathrm{~mm}, \theta=7 \mathrm{~mm}$, $H=1.38, C_{f}=0.00418, R_{\theta}=950$, for a free-stream velocity, $U_{\infty}$, of $2.1 \mathrm{~m} / \mathrm{s}$.

The profile of the mean streamwise velocity $\bar{U}$, in both the perturbed and unperturbed flows, nondimensionalized by the wall friction velocity $u^{*}$ in the unperturbed boundary layer is shown in Fig. 2. Compared to the unperturbed boundary layer, the mean streamwise velocity is reduced throughout the region $y^{+}<30$ and more so the higher the frequency. Further from the wall, $\bar{U}$ is slightly larger in order to conserve mass flux and exhibits a well defined log law. The mean velocity gradient $\partial \bar{U} / \partial y$ is increased for $y^{+}>8$ and decreased below this position.

A summary of the one-point statistics of the flow with spanwise oscillations, $f^{+}=0.01$, and a wall speed

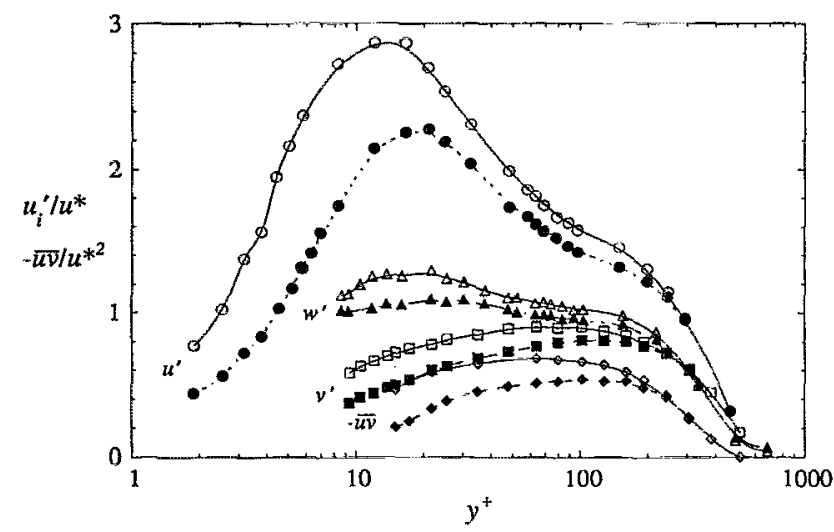

FIG. 3. RMS profiles of the three fluctuating velocity components and Reynolds stress $\overrightarrow{u v}$ for $f^{+}=0.01$ compared with the unperturbed boundary layer (open symbols).
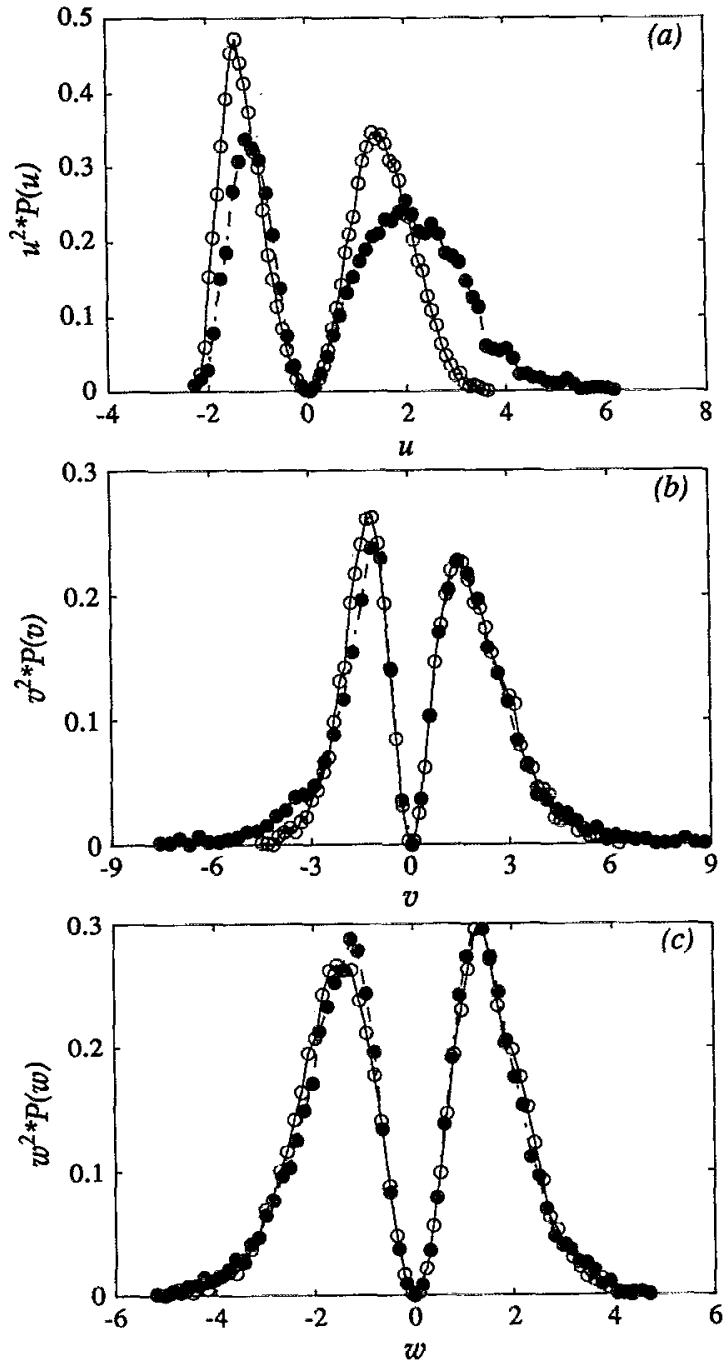

FIG. 4. Weighted probability density for (a) $u$, (b) $v$, and (c) $w$ at $y^{+}=9$ and $f^{+}=0.01$, compared to the unperturbed boundary layer (open symbols).

$W_{w}=0.45 U_{\infty} \sin \omega t$, is shown in Fig. 3. All statistical measures $\left(u^{\prime}, v^{\prime}, w^{\prime}\right.$ and $\left.-\overline{u v}\right)$, normalized by the wall friction velocity in the unperturbed boundary layer, are considerably reduced when $y^{+}<200$. Results for other frequencies exhibit qualitatively similar behavior. Note that the relative reduction in $u^{\prime}(45 \%)$ and in $-\overline{u v}(50 \%)$ is larger than in $v^{\prime}$ $(34 \%)$ and $w^{\prime}(16 \%)$. The drop in turbulent kinetic energy is therefore mostly caused by a reduction of $u^{\prime}$ and $v^{\prime}$. A link between the variation of $u^{\prime}, v^{\prime}$ and $-\overline{u v}$ is clearly suggested by their corresponding transport equations. This also agrees qualitatively with the numerical results of Moin et al. ${ }^{2}$

The weighted probability density functions (PDFs) of $u$, $v$ and $w$, normalized so that $\int_{-\infty}^{+\infty} u_{i}^{2} P\left(u_{i}\right) d u_{i}=1$, are shown in Fig. 4. This representation does not show the significant weakening of the fluctuations for the 3-D flow, but it can provide useful information since changes in statistical quantities are related to changes in the flow structure.

It can be seen that the peaks in the PDFs of $u$ and $v$ are reduced, both for positive and negative fluctuations, but that the PDFs are increased for higher positive streamwise veloc- 




FIG. 5. The nondimensional dissipation length scales for $f^{+}=0.01$ compared to the unperturbed boundary layer (open symbols).

ity, $u$, and negative normal velocity, $v$, whereas there is no change in the PDF of $w$. The spanwise oscillations do not affect kinematic events such as ejections and sweeps in the same manner. The contribution of $u>0$ and $v<0$ events to turbulence production is increased in the 3-D case, corresponding to the increasing importance of sweeps. Consequently future work should be focused on detailed conditional averaged fields in order to highlight these changes in the oscillatory flow structure.

In addition to a reduction in turbulent quantities, the fine structure of turbulence is affected by a spanwise oscillation. This is obvious from the behavior of the distribution of Taylor's microscales given by $\lambda_{11}^{(i)^{+}}=u^{*} / \nu \sqrt{\overline{u^{2}} / \overline{\left(\partial u / \partial x_{i}\right)^{2}}}$ (here $x_{1}=x$ and $\left.x_{2}=y\right)$. Figure 5 shows the profiles of $\lambda_{11}^{(1)^{+}}$and $\lambda_{11}^{(2)^{+}}$, as functions of $y^{+}$, for an unperturbed boundary layer and for one oscillating at a frequency $f^{+}=0.01$. Both dissipation length scales are reduced for $y^{+}<40$, with a greater reduction in $\lambda_{11}^{(1)^{+}}$, corresponding to an increase in $\frac{(\partial u / \partial x)^{2}}{}$ and a reduction in $\overline{(\partial u / \partial y)^{2}}$.

Another significant change is the appreciable reduction of the correlation coefficient between the normal and the streamwise gradient of the longitudinal fluctuation $u$ (in the region $y^{+}<30$ ) (Fig. 6). The correlation between these two

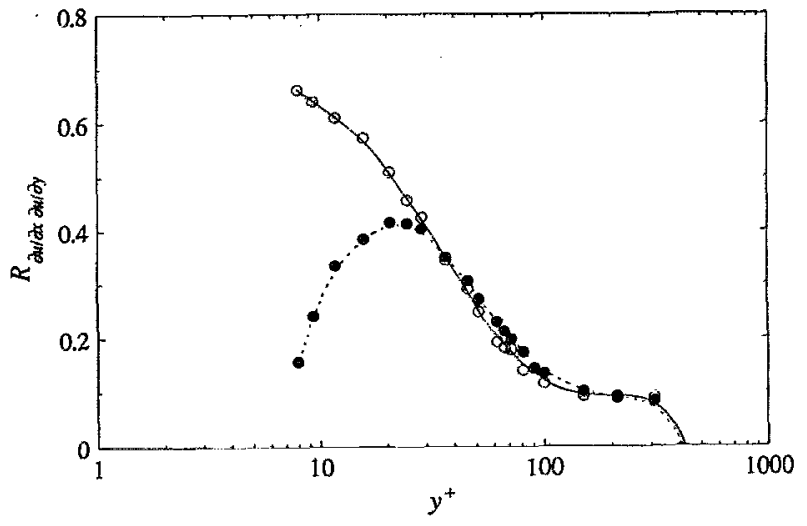

FIG. 6. Correlation coefficient between the normal and longitudinal streamwise velocity gradients at $f^{+}=0.01$ and for the unperturbed boundary layer (open symbols).

gradients is related to the sloping near-wall shear layers which are located at the interfaces between upstream highspeed fluid and downstream low-speed fluid. Johansson et al..$^{5}$ have shown that these near-wall shear layers play an important part in turbulence production. This reduction suggests that the near-wall shear layer of the 3-D flow and that of the unperturbed flow do not have the same direction. This mechanism can therefore be easily studied by two-point correlations in order to acquire information on there orientation of the shear layer in the 3-D flow.

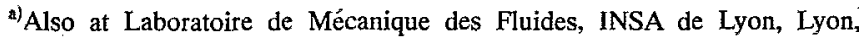
France.

${ }^{1} \mathrm{P}$. Bradshaw and N. Pontikos, "Measurements in a turbulent boundary layer on an infinite swept wing," J. Fluid Mech. 159, 105 (1985).

${ }^{2}$ P. Moin, T.-H. Shih, D. Driver, and N. N. Mansour, "Direct numerical simulation of a three-dimensional turbulent boundary layer," Phys. Fluids A 2, 1846 (1990).

${ }^{3} \mathrm{O}$. Sendstad and P. Moin, "On the mechanics of 3-D turbulent boundary layer," in Proceedings of the Eighth Symposium on Turbulent Shear Flows (Technical University of Munich, Germany, 1991), Vol. 1, p. 5-4-1.

${ }^{4}$ W. J. Jung, N. Mangiavacchi, and R. Akhavan, "Suppression of turbulence in wall-bounded flows by high-frequency spanwise oscillations," Phys. Fluids A 4, 1605 (1992).

${ }^{5}$ A. V. Johansson, P. H. Alfredsson, and J. Kim, "Evolution and dynamics of shear-layer structures in near-wall turbulence," J. Fluid Mech. 224, 579 (1991). 